\title{
Characteristics of upper extremity's muscle strength in Turkish national wheelchair basketball players team
}

\author{
Bihter Akınoğlu1,*, Tuğba Kocahan² \\ 'Department of Physiotherapy and Rehabilitation, Faculty of Health Sciences, Ankara Yıldırım Beyazıt University, Ankara, Turkey \\ ${ }^{2}$ Department of Health Services, The Ministry of Youth and Sports, Sports General Directorship, Center of Athlete Training and Health Research, Ankara, Turkey
}

The objective of this study was to reveal characteristics of muscle strength of upper extremities of wheelchair (WC) basketball players and to ensure more-specific training program preparation. Isokinetic muscle strength of 12 WC basketball players were assessed by ISOMED 2000 device. The assessment protocol was evaluated at $60^{\circ} / \mathrm{sec}$ velocity with 5 times repeated force and at $240 \% \mathrm{sec}$ with 15 times repeated force. This protocol was carried out individually for shoulder flexion-extension and wrist flexion-extension movements at the right and left extremities. The flexion/extension ratio was determined to be outside of the ratios accepted as normal for primarily shoulder joint and for wrist joint. The extension movement was stronger than flexion movement in the shoulders at both velocities and the flexion movement was stronger than extension movement in the wrist. The repeat times where the peak torque occurred were $2-3$ repeats at $60^{\circ} / \mathrm{sec}$ velocity during flexion and extension movements for the wrist and shoulders, and the peak torque occurred at an average of 5-6 repeats in the shoulders at $240 \% \mathrm{sec}$ velocity and it occurred at 3-4 repeats in the wrist. The angles where the peak torque of the shoulder flexion and extension occurred varied between $80^{\circ}-115^{\circ}$ at both velocities, and it varied between $5^{\circ}-30^{\circ}$ angles for the wrist. As this study revealed, determination of muscle strength characteristics of WC athletes and especially using objective isokinetic devices will guide the planning of the appropriate training and exercise programs and preventing sports injuries in long term.

Keywords: Disabled sports, Isokinetic, Physical therapy, Sport physiotherapy, Wheelchair basketball

\section{INTRODUCTION}

Competitive sports for disabled individuals have entered into a fast development process during the recent years. These sports have gained a place in a wide spectrum reaching open athletes and elite athletes. Paralympic games have raised awareness for the participation of disabled individuals in these sports. These games form an exercise characteristic for preventive health and improve cardio-metabolic aptness. Furthermore, they develop social integration of the disabled individuals by improving their self-confidence, self-competency and life quality (Blauwet and Willick, 2012). Wheelchair (WC) basketball is the most popular paralympic sport (Gil-Agudo et al., 2010). This sport consists of activities demanding explosive strength and speed and intermediate intensity (Ozmen et al., 2014).
WC-use requires the active and coordinated usage of upper extremity muscles especially shoulder complex muscles actively and coordinately. The shoulder flexors make the major contribution during WC-pushing stage and the shoulder extensors are dominant during the return stage. There is increased shoulder muscle cocontraction seen during the passage stage between these two stages (Morrow et al., 2009; Rankin et al., 2011).

The upper extremity injuries are seen in WC-using individuals considerably (Boninger et al., 1998). Shoulder pain is the most frequently seen complaint (Fullerton et al., 2003). Finley and Rodgers (2004) reported in their study conducted on WC-using individuals that the persons suffered shoulder pain caused by many reasons including biceps tendinitis and shoulder instability, and $29 \%$ of these people had pain during relaxation as well. Curtis and Black (1999) showed in their study that $90 \%$ of women
*Corresponding author: Bihter Akınoğlu (iD http://orcid.org/0000-0002-8214-7895 Department of Physiotherapy and Rehabilitation, Faculty of Health Sciences, Ankara Yildırım Beyazit University, Ankara, Turkey

Tel: +90-0505-938-41-36 Fax: +90-0312-305-15-15, E-mail: rgkardelen@yahoo.com Received: December 6, 2016 / Accepted: February 1, 2017
This is an Open Access article distributed under the terms of the Creative Commons Attribution Non-Commercial License (http://creativecommons.org/licenses/by-nc/4.0/) which permits unrestricted non-commercial use, distribution, and reproduction in any medium, provided the original work is properly cited. 
WC basketball athletes had shoulder and upper extremity pain. Muscle strength unbalance in the shoulder region and relative weakness in the depressors of humeral head can be affective for the development and continuation of rotator cuff compression syndrome (Burnham et al., 1993). In addition to shoulder complexities, elbow and wrist injuries occur frequently in WC athletes. Wrist extension and wrist joint movement speed with deviations are the factors affecting dynamic performance and causing injuries (Lee et al., 2016). Both wrist extension and flexion movement gap and muscle strength are the factors affecting performance of WC basketball players (Wang et al., 2005).

High level of condition is required for winning a competition in WC basketball like in all sports. The major factors affecting WC basketball performance are muscle strength and sprint speed. Having high level of muscle strength in WC basketball will ensure both performance improvement in basic movements unique to basketball and independence in daily life activities. The objective of this study was to reveal the isokinetic muscle strength characteristics of upper extremity in WC basketball athletes. The descriptive information will lead to the description of injury-preventing programs and more specific training programs.

\section{MATERIALS AND METHODS}

This study was conducted to reveal upper extremity isokinetic muscle strength characteristics in WC basketball players at the Ministry of Youth and Sports, Sports General Directorship, Health Affairs Department Directorship (Athlete Training and Health Research Center). All the athletes who accepted to participate in the study were informed about the study purpose, the assessments contained in the study and the benefits of the study before starting the study and the study was based on volunteerism, and approval was obtained. The necessary permit and approval was obtained from the Ethics Committee of Ankara Yıldırım Beyazit University to conduct the study (13/03 [346]). The participants in the present study consisted of 12 male WC basketball players, aged 23- 40 years, who are members of the Turkish national WC basketball team and their scores ranged between 2.54.5. The descriptive characteristics, age, height, weight and disability types of the athletes were recorded.

\section{Isokinetic muscle strength assessment}

Isokinetic muscle strength was assessed by ISOMED 2000 (D. $\&$ R. Ferstl GmbH, Hemau, Germany) device. Prior to the test, the athletes did general and special warming workouts for 10 min. Following the warming, the athletes were taken to the isokinetic device one by one for measurement and the device was adjusted according to their individual anthropometric structures. During the test, their weights were entered in the computer and the program was set. The suitability of the movement range of the joint to be tested to the angles that are to be tested was determined by making the athletes do a sample movement at a very low speed. At the same time, the gravity effect was set to zero. The joint angles were adjusted in the assessment by considering the joint movement ranges of the persons and the measurement characteristics of the device. An assessment was made for the shoulder flexion/extension movement between the angles of $30^{\circ}$ flexion and $120^{\circ}$ flexion, and for the wrist flexion/extension movement between the angles of $50^{\circ}$ flexion and $60^{\circ}$ extension. The assessment protocol: the athletes warmed by doing the flexion/extension movement at $90 \%$ sec with five repeats as submaximal and they were ensured to perceive the movement. Following the warming movement and a 30-sec rest, they did maximal flexion/extension movement at $60 \%$ sec speed and with five repeats (Kim and Jeong, 2016) and again following a 30-sec rest, they did maximal flexion/extension movement at $240 \%$ sec speed and with 15 repeats and the test was completed. The assessments were made bilaterally for each joint and first the dominant side was assessed and 3 min later the nondominant side was assessed. The same protocol was applied the following day for the wrist joint.

\section{Statistical analysis}

The data collected regarding the isokinetic strength of the athletes were analyzed by IBM SPSS Statistics ver. 22.0 (IBM Co., Armonk, NY, USA). The descriptive statistics of all variables were determined. The results were indicated as mean \pm standard deviation.

\section{RESULTS}

Demographic characteristics and disability types of the athletes are shown in Table 1.

The peak torque values and peak torque/weight values of dominant - nondominant shoulder flexion and extension movements of the athletes and wrist flexion and extension movements at $60 \% \mathrm{sec}$ and $240 \%$ sec velocity, and the angles of these values and repeat times are shown in Table 2.

The extension peak torque was found to be higher than the flexion peak torque in the shoulder joint, and the flexion peak torque value was higher in the wrist. The peak torque values were generated with 2-3 repeats at $60 \%$ sec velocity at both movements 
for the wrist and shoulder. It occurred at $240 \%$ sec velocity with an average of 5-6 repeats for both movements, and it occurred at 3-4 repeats for the wrist. Considering the angle values where the peak torque value is generated, it was at $60^{\circ} / \mathrm{sec}$ velocity in the shoulder joint as similar in the dominant and nondominant sides approximately $81^{\circ}-86^{\circ}$ and $112^{\circ}-109^{\circ}$ for flexion and extension respectively. At $240^{\circ} / \mathrm{sec}$ velocity, it was $110^{\circ}-97^{\circ} ; 108^{\circ}-106^{\circ}$. For the wrist, at $60^{\circ} / \mathrm{sec}$ velocity, it was approximately $16^{\circ}-24^{\circ}$ at

Table 1. Demographic characteristics of the athletes who participated in the study

\begin{tabular}{lc}
\hline Characteristic & Value \\
\hline Age $(\mathrm{yr})$ & $28.91 \pm 5.00$ \\
Height $(\mathrm{cm})$ & $75.64 \pm 11.79$ \\
Weight $(\mathrm{kg})$ & $72.73 \pm 10.2$ \\
Body mass index $\left(\mathrm{kg} / \mathrm{m}^{2}\right)$ & $30.44 \pm 8.08$ \\
Disability type & \\
Spina Bifida & 1 \\
Polyomyelitis & 3 \\
Amputation & 2 \\
Paraparesis & 2 \\
Paraplegia & 2 \\
Meningomyelocele & 1 \\
\hline
\end{tabular}

Values are presented as mean \pm standard deviation or number. flexion and at $5^{\circ}-6^{\circ}$ extension as similar in the dominant and nondominant sides respectively for the flexion and extension; at $240^{\circ}$, for flexion and extension, it was respectively $22^{\circ}-25^{\circ}$ flexion and $13^{\circ}-6^{\circ}$ extension.

The percentages of the shoulder and wrist flexion/extension movements of the athletes at $60^{\circ} / \mathrm{sec}$ and $240 \% \mathrm{sec}$ velocity for the dominant and nondominant sides and between the right and left, and the standard deviation values are shown in Table 3.

The extension isokinetic muscle strength in the shoulder joint was higher than the flexion isokinetic muscle strength, and the flexion isokinetic muscle strength in the wrist was higher than the extension isokinetic muscle strength (Table 3).

\section{DISCUSSION}

In this study, the peak torque, peak torque/kg, right/left ration percentage and flexion/extension ratio percentage of WC basketball athletes were determined.

There are two studies in the literature assessing isokinetic muscle strength in healthy individuals. Danneskiold-Samsoe et al. (2009) categorized according to age and gender and measured the wrist flexion and extension and shoulder flexion and extension at $60 \%$ sec velocity, and Harbo et al. (2012) published two studies

Table 2. Peak torque, peak torque/weight, repeat times, and angle values of wheelchair basketball athletes for shoulder and wrist flexion-extension movement at $60 \%$ sec and $240 \%$ sec velocity

\begin{tabular}{|c|c|c|c|c|}
\hline \multirow{2}{*}{ Angular velocity } & \multicolumn{2}{|c|}{ Dominant } & \multicolumn{2}{|c|}{ Nondominant } \\
\hline & Flexion & Extension & Flexion & Extension \\
\hline \multicolumn{5}{|l|}{ Shoulder } \\
\hline $60^{\circ} / \mathrm{sec}$ (peak torque) (N/m) & $109.63 \pm 28.58$ & $150.71 \pm 38.75$ & $101.15 \pm 28.47$ & $146.98 \pm 36.07$ \\
\hline $60^{\circ} / \mathrm{sec}$ (peak torque/weight) ( $\mathrm{N} / \mathrm{kg}$ ) & $13.87 \pm 42.77$ & $17.52 \pm 53.43$ & $13.75 \pm 42.81$ & $22.23 \pm 70.02$ \\
\hline Angle $\left({ }^{\circ}\right)$ & $81.66 \pm 14.36$ & $112.16 \pm 16.44$ & $86.16 \pm 15.39$ & $109.75 \pm 15.12$ \\
\hline Repeat number & $2.33 \pm 1.49$ & $2.91 \pm 1.44$ & $3.33 \pm 1.49$ & $3.00 \pm 1.20$ \\
\hline $240^{\circ} / \mathrm{sec}$ (peak torque) $(\mathrm{N} / \mathrm{m})$ & $93.83 \pm 29.64$ & $130.22 \pm 36.93$ & $84.31 \pm 26.37$ & $119.61 \pm 4.34$ \\
\hline $240^{\circ} / \mathrm{sec}$ (peak torque/weight) (N/kg) & $11.09 \pm 33.92$ & $15.6 \pm 47.8$ & $12.37 \pm 38.9$ & $17.11 \pm 53.56$ \\
\hline Angle $\left({ }^{\circ}\right)$ & $110.75 \pm 64.88$ & $108.16 \pm 10.02$ & $97.58 \pm 16.37$ & $106.58 \pm 6.81$ \\
\hline Repeat number & $6.75 \pm 4.88$ & $6.66 \pm 3.62$ & $6.41 \pm 3.70$ & $4.75 \pm 3.74$ \\
\hline \multicolumn{5}{|l|}{ Wrist } \\
\hline $60^{\circ} / \mathrm{sec}$ (peak torque) (N/m) & $32.45 \pm 7.96$ & $12.74 \pm 3.19$ & $35.75 \pm 7.96$ & $13.54 \pm 3.23$ \\
\hline $60^{\circ} / \mathrm{sec}$ (peak torque/weight) ( $\mathrm{N} / \mathrm{kg}$ ) & $0.44 \pm 0.09$ & $0.17 \pm 0.04$ & $0.50 \pm 0.13$ & $0.18 \pm 0.05$ \\
\hline Angle $\left({ }^{\circ}\right)$ & $16.91 \pm 20.41$ & $-6.91 \pm 16.15$ & $24.41 \pm 11.88$ & $-5.25 \pm 19.7$ \\
\hline Repeat number & $2.75 \pm 1.54$ & $2.41 \pm 1.31$ & $3.00 \pm 1.41$ & $3.25 \pm 1.28$ \\
\hline $240^{\circ} / \mathrm{sec}$ (peak torque) $(\mathrm{N} / \mathrm{m})$ & $24.15 \pm 3.35$ & $12.10 \pm 9.24$ & $26.39 \pm 6.78$ & $13.30 \pm 8.24$ \\
\hline $240^{\circ} / \mathrm{sec}$ (peak torque/weight) (N/kg) & $0.33 \pm 0.04$ & $0.12 \pm 0.02$ & $0.36 \pm 0.09$ & $0.11 \pm 0.03$ \\
\hline Angle $\left({ }^{\circ}\right)$ & $22.83 \pm 9.61$ & $-13.66 \pm 18.69$ & $25.50 \pm 8.09$ & $-6.91 \pm 31.16$ \\
\hline Repeat number & $4.08 \pm 3.94$ & $4.50 \pm 3.94$ & $3.66 \pm 2.66$ & $3.16 \pm 1.26$ \\
\hline
\end{tabular}

Values are presented as mean \pm standard deviation. 
Table 3. The percentage between the right and left for the shoulder and wrist flexion and extension movements and the percentage of flexion/extension for all movements

\begin{tabular}{|c|c|c|c|c|}
\hline \multirow{2}{*}{ Joint } & \multicolumn{2}{|c|}{ Right/left ratio (\%) } & \multicolumn{2}{|c|}{ Flexion/extension ratio (\%) } \\
\hline & Flexion & Extension & Dominant & Nondominant \\
\hline \multicolumn{5}{|c|}{ Shoulder joint } \\
\hline $60^{\circ} / \mathrm{sec}$ & $109.1 \pm 18.8$ & $103.2 \pm 21.3$ & $73.28 \pm 9.21$ & $69.12 \pm 9.63$ \\
\hline $240^{\circ} / \mathrm{sec}$ & $113.1 \pm 31.1$ & $106.6 \pm 22.4$ & $73.26 \pm 13.6$ & $70.2 \pm 13.3$ \\
\hline \multicolumn{5}{|l|}{ Wrist joint } \\
\hline $60^{\circ} / \mathrm{sec}$ & $93.86 \pm 26.57$ & $97.65 \pm 27.99$ & $260.28 \pm 52.78$ & $267.55 \pm 54.21$ \\
\hline $240^{\circ} / \mathrm{sec}$ & $96.17 \pm 23.71$ & $119.32 \pm 29.71$ & $267.11 \pm 50.08$ & $331.48 \pm 72.99$ \\
\hline
\end{tabular}

Values are presented as mean \pm standard deviation.

Table 4. Literature values of the shoulder and wrist flexion and extension peak torque at $60^{\circ} / \mathrm{sec}$ velocity

\begin{tabular}{|c|c|c|c|c|}
\hline \multirow{2}{*}{ Joint angular velocity } & \multicolumn{2}{|c|}{ Dominant } & \multicolumn{2}{|c|}{ Nondominant } \\
\hline & Flexion & Extension & Flexion & Extension \\
\hline \multicolumn{5}{|l|}{ Shoulder } \\
\hline $60^{\circ} / \mathrm{sec}$ (our study) & $109.63 \pm 28.58$ & $150.71 \pm 38.75$ & $101.15 \pm 28.47$ & $146.98 \pm 36.07$ \\
\hline $60^{\circ} / \sec$ (Moon et al. 2013) & $51.4 \pm 13.9$ & $75.2 \pm 20.1$ & $41.4 \pm 14.4$ & $68.3 \pm 21.2$ \\
\hline \multicolumn{5}{|c|}{$60^{\circ} / \mathrm{sec}$ (Danneskiold-Samsøe et al. 2009) } \\
\hline $20-29$ yr & $51.8 \pm 10.6$ & $72.1 \pm 19.5$ & & \\
\hline $30-39$ yr & $54.7 \pm 7.3$ & $65.6 \pm 15.1$ & & \\
\hline \multicolumn{5}{|l|}{ Wrist } \\
\hline $60^{\circ} / \mathrm{sec}$ (our study) & $32.45 \pm 7.96$ & $12.74 \pm 3.19$ & $35.75 \pm 7.96$ & $13.54 \pm 3.23$ \\
\hline \multicolumn{5}{|c|}{$60^{\circ} / \mathrm{sec}$ (Danneskiold-Samsøe et al. 2009) } \\
\hline $20-29 \mathrm{yr}$ & $20.6 \pm 4.4$ & $10.4 \pm 3.3$ & & \\
\hline $30-39$ yr & $19.3 \pm 4.7$ & $11.0 \pm 1.2$ & & \\
\hline
\end{tabular}

determining isokinetic muscle strength in 178 healthy individuals at angular velocities that were different than ours (Danneskiold-Samsoe et al., 2009; Harbo et al., 2012). Moreover, Moon et al. (2013) published a study evaluating isokinetic muscle strength in WC tennis athletes as similar to our study. Our values at $60 \%$ sec, and the flexion and extension peak torque values for the dominant and nondominant sides of Moon et al. (2013), and the shoulder and wrist flexion and extension peak torque values for 20-29 age group and 30-39 age group of Danneskiold-Samsoe et al. (2009) are shown in Table 4.

The peak torque of the shoulder flexion and extension in WC basketball athletes included in our study was approximately 2 times of those of the healthy participants in Danneskiold-Samsoe et al. (2009) study and also of WC tennis athletes in Moon et al. (2013) study. It is thought that this outcome is caused by the fact that WC basketball sports requires intense upper extremity muscle strength for coordinated movement of WC and ball, and these athletes are included in the Paralympic class. Danneskiold-Samsoe et al. (2009) found in their study that the wrist flexion/extension ratio was close to 1 in healthy individuals, and in our study, it was deter- mined to be almost $1 / 3$ in the favor of the flexors. Despite a high wrist flexor strength seems to be in favor of the athletes; it must be remembered that the muscle strength imbalance between the wrist flexors and extensors will be a preparatory factor for sports injuries. It was seen that the athletes had tennis player elbow complaint in their backgrounds as parallel to our results. We think that providing information on this issue to athletes and health team, and establishment of training programs for strengthening wrist extensors will prevent overuse injuries. There are numerous studies in the literature showing that individuals become prone to injuries due to muscle imbalances (Blauwet and Willick, 2012; Knight, 1980). Since there were only the peak torque data in Danneskiold-Samsoe et al. (2009) study, peak torque/kg and other characteristic data were not able to be compared. Isokinetic assessments vary according to angular velocity. Therefore, it is not possible to make a comparison with this study since Harbo et al. (2012) assessed the wrist in angular velocities different than ours. The flexion/extension difference and right and left difference in our study are shown in percentages in Table 3. These data are critical for the existence of a balance between agonist/antagonist muscle forces and the disrup- 
Table 5. The literature values of the comparisons of the right-left and flexion/extension for the shoulder joint at $60^{\circ} / \mathrm{sec}$ velocity

\begin{tabular}{lrrrrr}
\hline \multirow{2}{*}{ Shoulder joint } & \multicolumn{2}{c}{ Right/left ratio $(\%)$} & & \multicolumn{2}{c}{ Flexion/extension ratio (\%) } \\
\cline { 2 - 5 } \cline { 5 - 6 } & Flexion & Extension & Dominant & Nondominant \\
\hline $60^{\circ} / \mathrm{sec}$ (our study) & $109.1 \pm 18.8$ & $103.2 \pm 21.3$ & $73.28 \pm 9.21$ & $69.12 \pm 9.63$ \\
$60^{\circ} / \mathrm{sec}$ (Moon et al. 2013) & $79.1 \pm 12.3$ & $90.0 \pm 10.6$ & & $149.0 \pm 23.1$ & $173.7 \pm 46.0$ \\
\hline
\end{tabular}

tion of the balance make the person prone to injuries (Knapik et al., 1991). Perrin (1993) found this ratio as 75\%-85\% for shoulder flexion and extension in their study. In our study, this ratio was found as approximately $71.2 \%$ as parallel to this result.

Hughes et al. (1999) assessed the antagonist/agonist ratio between the wrist flexion and extension in their study and concluded that there was not a significant difference between the dominant and nondominant sides. In our study, we found that the wrist flexion/extension percentage in both sides varied between $260 \%-331 \%$. The difference was outside of the normal limits and we suggest that the wrist extensors are strengthened and muscle imbalance is overcome to prevent sports injuries due to muscle strength imbalance.

Kannus (1994) reported in their study on isokinetic assessment that more than $10 \%$ of difference between the right and left extremities was abnormal. In our study, it was determined that the difference between the right and left side at the shoulder flexion and wrist extension at $240 \%$ sec velocity was more than $10 \%$. Therefore, workout programs must be added to the training programs to improve the strength for right and left bilaterally in activities requiring speed for especially the shoulder flexion and wrist extension. This puts forward the significance of neuro-reactive workouts in the training programs.

The results of our study and the percentages between the rightleft and extension/flexion in Moon et al. (2013) study conducted on WC tennis players are shown in Table 5. It was seen that the percentage of the right-left flexion and the percentage of the dominant side flexion/extension in WC tennis players were higher than those of the basketball players included in our study. We think that this difference is caused by the inclusion of WC using athletes by both groups however tennis is more asymmetric in comparison to basketball.

In addition, in our study the repeat number and angles where the peak strength occurs are indicated. In our study, there were athletes reaching the peak torque in the first repeat, there were also athletes reaching the torque strength at the 14 th repeat. The peak occurred at $60 \%$ sec velocity for the shoulder and wrist flexion and extension at an average of 2-3 repeats; and the peak occurred at $240 \%$ sec for the shoulder flexion and extension at the fifth-sixth repeat, and it occurred at the third-fourth repeat for the wrist flexion and extension. This outcome of our study shows that the repeat number must be increased as the assessment speed increases. Studies need to be conducted on this issue with high number of athletes.

It was determined in our study that the peak torque occurred at the angles when the shoulder was in an elevated position and at the angles when the wrist was at a functional holding position. We think that this angle was affected by the fact that the athletes were basketball players and basketball throwing position affected the angle for peak torque generation in the wrist. In this context, the angles where the peak torque is generated should be considered when training programs are established.

The limitations of our study were failure to compare the peak torque values according to the disability type and age due to the low number of athletes.

In conclusion, WC basketball sport is the most popular one among paralympic sports branches and in Turkey. It is a critical tool for the rehabilitation of disabled persons and their integration in society. WC basketball sport is exercised at a professional level in the world and Turkey. Prevention of sports injuries is important in WC basketball like in other sports branches. The workout and training programs is an issue that needs attention for both prevention of sports injuries and achieving high performance in this sports branch. As our study revealed, determination of characteristics of muscle strength of WC athletes and especially using objective isokinetic tools will be guiding for the training program planning and prevention of sports injuries in long term.

\section{CONFLICT OF INTEREST}

No potential conflict of interest relevant to this article was reported.

\section{REFERENCES}

Blauwet C, Willick SE. The paralympic movement: using sports to promote health, disability rights, and social integration for athletes with disabilities. PM R 2012;4:851-856. 
Boninger ML, Cooper RA, Shimada SD, Rudy TE. Shoulder and elbow motion during two speeds of wheelchair propulsion: a description using a local coordinate system. Spinal Cord 1998;36:418-426.

Burnham RS, May L, Nelson E, Steadward R, Reid DC. Shoulder pain in wheelchair athletes. The role of muscle imbalance. Am J Sports Med 1993;21:238-242.

Curtis KA, Black K. Shoulder pain in female wheelchair basketball players. J Orthop Sports Phys Ther 1999;29:225-231.

Danneskiold-Samsøe B, Bartels EM, Bülow PM, Lund H, Stockmarr A, Holm CC, Wätjen I, Appleyard M, Bliddal H. Isokinetic and isometric muscle strength in a healthy population with special reference to age and gender. Acta Physiol (Oxf) 2009;197 Suppl 673:1-68.

Finley MA, Rodgers MM. Prevalence and identification of shoulder pathology in athletic and nonathletic wheelchair users with shoulder pain: a pilot study. J Rehabil Res Dev 2004;41(3B):395-402.

Fullerton HD, Borckardt JJ, Alfano AP. Shoulder pain: a comparison of wheelchair athletes and nonathletic wheelchair users. Med Sci Sports Exerc 2003;35:1958-1961.

Gil-Agudo A, Del Ama-Espinosa A, Crespo-Ruiz B. Wheelchair basketball quantification. Phys Med Rehabil Clin N Am 2010;21:141-156.

Harbo T, Brincks J, Andersen H. Maximal isokinetic and isometric muscle strength of major muscle groups related to age, body mass, height, and sex in 178 healthy subjects. Eur J Appl Physiol 2012;112:267-275.

Hughes RE, Johnson ME, O'Driscoll SW, An KN. Normative values of agonist-antagonist shoulder strength ratios of adults aged 20 to 78 years. Arch Phys Med Rehabil 1999;80:1324-1326.

Kannus P. Isokinetic evaluation of muscular performance: implications for muscle testing and rehabilitation. Int J Sports Med 1994;15 Suppl
1:S11-18.

Kim CG, Jeoung BJ. Assessment of isokinetic muscle function in Korea male volleyball athletes. J Exerc Rehabil 2016;12:429-437.

Knapik JJ, Bauman CL, Jones BH, Harris JM, Vaughan L. Preseason strength and flexibility imbalances associated with athletic injuries in female collegiate athletes. Am J Sports Med 1991;19:76-81.

Knight KL. Strength imbalance and knee injury. Phys Sportsmed 1980; 8:140.

Lee SY, Chieh HF, Lin CJ, Jou IM, Kuo LC, Su FC. The potential risk factors relevant to lateral epicondylitis by wrist coupling posture. PLoS One 2016;11:e0155379.

Moon HB, Park SJ, Kim AC, Jang JH. Characteristics of upper limb muscular strength in male wheelchair tennis players. J Exerc Rehabil 2013; 9:375-380.

Morrow MM, Hurd WJ, Kaufman KR, An KN. Upper-limb joint kinetics expression during wheelchair propulsion. J Rehabil Res Dev 2009;46: 939-944.

Ozmen T, Yuktasir B, Yildirim NU, Yalcin B, Willems ME. Explosive strength training improves speed and agility in wheelchair basketball athletes. Rev Bras Med Esporte 2014;20:97-100.

Perrin DH. Isokinetic exercise and assessment. Champain (IL): Human Kinetics Publishers; 1993.

Rankin JW, Richter WM, Neptune RR. Individual muscle contributions to push and recovery subtasks during wheelchair propulsion. J Biomech 2011;44:1246-1252.

Wang YT, Chen S, Limroongreungrat W, Change LS. Contributions of selected fundamental factors to wheelchair basketball performance. Med Sci Sports Exerc 2005;37:130-137. 